\title{
Huiles et corps gras des Pays du Sud et lipochimie
}

\author{
Jérôme LECOMTE \\ Michel PINA \\ Pierre VILLENEUVE \\ CIRAD, \\ UMR IATE, \\ F-34398 Montpellier, \\ France \\ $<$ jerome.lecomte@cirad.fr $>$
}

Article reçu le 6 octobre 2012

Accepté le 10 décembre 2012

\begin{abstract}
Oils and fats from southern countries for oleochemistry
During the last decades the oleochemistry has gradually flooded our everyday life through a mass of products and applications. This is mainly due to the wide structural diversity and reactivity of fatty acids and the growing demand for bio-sourced goods. Today, almost one quarter of the global vegetable oil production is dedicated to nonfood applications, the contribution of animal fats being much more modest. Excepted biodiesels, the chemical applications of tropical edible oils depend on their fatty acids composition: lauric oils (coconut and palm kernel) and palm stearin will be mostly converted into surface actives ingredients while unsaturated oils rather will be subjected to double bond functionalization or cracking. Alongside of major vegetable oils only a few non-edible tropical oils are exclusively intended to oleochemistry such as Castor and Jatropha. Some other non-food oilseed crops (Cuphea, Lesquerella, Vernonia, black mustard. . .) are promising but further researches are still needed for their development in Southern Countries. In the future, the production increase of major vegetable oils and the development of new ones will face many challenges relating to environmental issues, competition between food and non-food uses and between non-food applications themselves.
\end{abstract}

Key words: Southern Countries, oleochemistry, vegetable oil-based chemicals, nonfood uses, palm oil, global market

considère que le savon en est une des premières applications, sinon la première.

\section{Des premiers savons à la lipochimie moderne}

Il faut remonter à la Babylone antique, il y a plus de 4200 ans, pour trouver des descriptions d'une préparation à base de cendres de bois et d'huiles végétales. Bien qu'il ne correspondît pas véritablement à un savon, stricto sensu, ce mélange devait présenter néanmoins un certain pouvoir détergent au vu de son emploi pour le dégraissage de la laine et des tissus avant teinture. La fabrication et I'utilisation du savon en tant que tel est beaucoup plus récente et vraisemblablement contemporaine du début de l'ère chrétienne, à en juger par les écrits de Pline I'Ancien, Galien et Arétée de Cappadoce (Gibbs, 1939). Au cours des siècles qui suivent, les procédés de fabrication et la qualité des savons ne cessent de s'améliorer avec la création de guildes d'artisans savonniers et un développement important des savons nobles à base d'huile d'olive sur le pourtour de la Méditerranée, notamment à Marseille au XVI ${ }^{e}$ siècle. II faut attendre néanmoins la fin du XVIII ${ }^{\mathrm{e}}$ siècle pour qu'un pas décisif vers la fabrication industrielle des savons soit franchi. Ainsi, en 1791, Nicolas Leblanc brevète un procédé révolutionnaire de synthèse du carbonate de sodium à partir d'une source abondante de chlorure de sodium, le sel marin. Quelques années plus tard en 1823, MichelEugène Chevreul pose les bases de la chimie des lipides, notamment la réaction de saponification, dans son ouvrage Recherches chimiques sur les corps gras d'origine animale. Enfin, Ernest Solvay met au point en 1861 un nouveau procédé de synthèse du vie quotidienne, la lipochimie a pourtant une origine fort ancienne si l'on 
carbonate de sodium à partir de sel marin, de calcaire et d'ammoniaque, procédé qui va rapidement supplanter celui de Leblanc, trop couteux et polluant (Taylor, 1957). D'une certaine manière, on peut considérer ces trois évènements majeurs comme fondateurs de la lipochimie moderne, science qui ne va cesser de se développer grâce aux apports constants de la chimie organique. Une des raisons de ce succès est la grande diversité des triglycérides et des acides gras qui les composent et, par conséquent, des applications potentielles. Ainsi, la longueur de la chaîne carbonée, la présence et la position d'une ou plusieurs double(s) liaison(s) éventuellement conjuguées - ainsi que l'existence de groupes fonctionnels latéraux (hydroxyle, carboxyle, amine, époxyde. ..) confèrent aux acides gras un large éventail de propriétés physicochimiques et de réactivités. Les transformations des triglycérides et des acides gras sont donc liées aux fonctions citées précédemment, aux travers de réactions chimiques classiques (Dumeignil, 2012) dont un rapide aperçu est donné ci-après :

(a) fonction ester des triglycérides: hydrolyse, saponification, transestérification, halogénation, réduction, amidation...

Produits obtenus : acides gras, savons, chlorures d'acyle, glycérides partiels, esters et alcools gras, sels d'amides ou d'amines, glycérol ;

(b) double liaison: hydrogénation, ozonolyse, méthatèse, halogénation, époxydation, polymérisation.
Produits obtenus : paraffines, oléfines, diacides, huiles fonctionnalisées, polymères ;

(c) hydroxyle latéral : deshydratation, pyrolyse, halogénation, estérification, formation d'uréthane (précurseur de polymères)...

Produits obtenus: huiles fonctionnalisées, polymères, acides sébacique et undécylénique, esters d'alkyles et d'arylalkyles.

Par ailleurs, d'autres facteurs importants ont contribué à l'essor de la lipochimie, et tout particulièrement ces dix dernières années, comme l'accroissement des contraintes énergétiques et environnementales, le développement de la chimie verte et des biotechnologies blanches, ainsi que l'évolution des demandes des consommateurs. Cependant, tout cela n'aurait été possible sans l'accès à des ressources abondantes et diversifiées telles que les huiles végétales et dans une moindre mesure les graisses animales. L'évolution de la lipochimie est donc intimement liée au marché des huiles végétales alimentaires, dont les Pays du Sud sont devenus les principaux acteurs.

\section{La production mondiale des huiles végétales alimentaires}

Avec un taux de croissance annuel de près de $5 \%$ depuis plusieurs années, le marché des huiles végétales alimentaires se montre particulièrement dyna- mique, comparé à celui des céréales et notamment du blé ( $\leq 2 \%$ p.a.). Comme raisons principales, citons l'occidentalisation des régimes alimentaires dans les pays émergents ou en développement et le remplacement progressif des graisses animales par les huiles végétales dans les pays développés. À cela, il faut aujourd'hui ajouter la part significative des applications non alimentaires des huiles végétales, avec en premier lieu les biocarburants.

En considérant les neuf principales huiles végétales alimentaires (USDAFAS, 2011) (palme, soja, colza, tournesol, palmiste, coton, arachide, coprah et olive), la production mondiale de l'année 2011 s'est élevée à environ 152 Mt (millions de tonnes). À elle seule, I'huile de palme en représente le tiers, suivie par les huiles de soja $(42,9 \mathrm{Mt}, 28,2 \%)$, colza $(22,8 \mathrm{Mt}$, $15,0 \%)$, tournesol (13,2 Mt, 8,6 \%), palmiste $(5,7 \mathrm{Mt}, 3,7 \%)$, coton $(5,4 \mathrm{Mt}$, 3,5\%), arachide (5,2 Mt, 3,4\%), coprah $(3,7 \mathrm{Mt}, 2,4 \%)$ et olive (3 Mt, $2 \%)$.

\section{Part des Pays du Sud dans le marché mondial des huiles végétales alimentaires}

Avec près de $60 \%$ de la production mondiale, les Pays du Sud (PDS) sont les principaux contributeurs. Ils fournissent en effet la totalité des huiles de palme, palmiste et coprah (60 Mt), $42 \%$ de

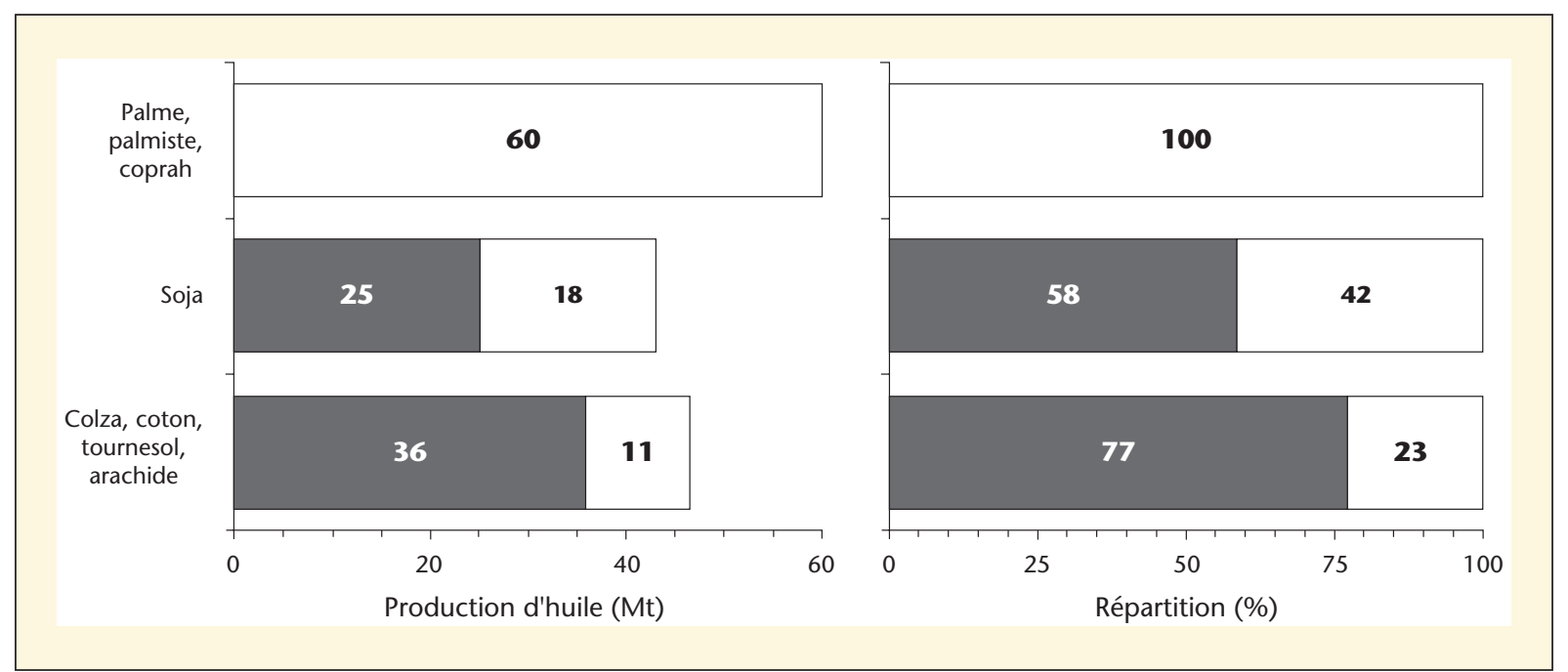

Figure 1. Contribution des Pays du Sud $(\square)$ et des autres pays $(\square)$ dans la production mondiale d'huiles végétales en 2011. 
I'huile de soja (18 Mt) et près d'un quart des autres huiles végétales alimentaires (figure 1). La répartition géographique de cette production n'est cependant pas homogène puisqu'elle concerne essentiellement deux grandes régions du monde, I'Amérique du Sud et l'Asie, et un nombre restreint de pays puisque près de $84 \%$ des huiles produites au Sud proviennent d'Argentine, du Brésil, $d^{\prime}$ Inde, de Malaisie et d'Indonésie. Ainsi, le marché des huiles de palme, palmiste et coprah, est très largement dominé par l'Indonésie et la Malaisie (50 Mt, $84 \%$ ) et celui du soja par l'Argentine et le Brésil (14,6 Mt, $82 \%)$. Bien que plus modeste, la production de I'Inde est davantage diversifiée puisqu'elle couvre $10 \%(1,7 \mathrm{Mt})$ du soja et $48 \%(5,1 \mathrm{Mt})$ des huiles de coton, tournesol, arachide et colza (figure 2). Enfin, on notera la faible contribution du continent africain avec à peine $6 \%$ des huiles produites dans les PDS et moins de $4 \%$ au niveau mondial.

Quel est le devenir de cette production du Sud? Si l'on s'intéresse à la consommation domestique ou, par opposition, au niveau d'exportation des trois groupes d'huiles produits par les cinq principaux pays cités précédemment, on observe des situations extrêmement contrastées. L'Indonésie et la Malaisie, par exemple, exportent les trois quarts de leur production d'huiles de palme, palmiste et coprah, le quart restant étant destiné à parts égales à des usages alimentaires et non-alimentaires. En revanche, dans le cas du Brésil et de I'Argentine, $62 \%$ de I'huile de soja produite est transformée sur place, principalement $(59 \%)$ en biocarbu- rants. Quant à I'Inde, dont l'impératif est de nourrir une population dépassant aujourd'hui les 1,2 milliard d'individus, la quasi-totalité de sa production d'huiles végétales est consommée dans le pays en tant qu'aliment de base.

\section{Évolution des usages non-alimentaires des huiles végétales}

Maintenue à un niveau relativement stable et modeste (à l'exception de I'huile de coprah) pendant des décennies, la part des usages nonalimentaires des huiles végétales (9 majeures), a subi une évolution spectaculaire au cours de la décennie 20012011 , en passant de $14 \%$ à $25 \%$. Ainsi, le quart de la production mondiale d'huiles végétales est aujourd'hui dédié à des applications autres que l'alimentation humaine.

Si la production de biodiesel, à partir de colza (Europe) ou de soja (Argentine, Brésil et aux États-Unis), représente une part significative de cette évolution (la production de biocarburant à partir de I'huile palme est encore relativement marginale), les applications non énergétiques y sont également pour beaucoup (tableau 1). Ainsi, la production de composés chimiques s'est particulièrement accrue en Malaisie, et dans une moindre mesure en Indonésie, à partir des huiles de palme et palmiste. Les usages non-alimentaires (essentiellement dans le secteur des tensioactifs) de l'huile de coprah, ont progressé plus modestement $(+12 \%)$, passant d'un niveau déjà élevé de $41 \%$ à $46 \%$.

\section{Huiles tropicales et lipochimie}

Dans le secteur de la lipochimie et hors biocarburants, deux types d'huile, l'un alimentaire et l'autre non, sont particulièrement importants en termes de volume ou d'applications. Le premier type concerne d'une part, les huiles riches en acides gras saturés, telles les huiles lauriques (palmiste et coprah) et les stéarines de palme, et d'autre part les huiles de soja et de palme (fractions oléiques) riches en insaturés. L'huile de ricin, quant à elle, représente le second type.

\section{Huiles lauriques et stéarines de palme}

Les huiles de palmiste et coprah, dites lauriques, se caractérisent par des teneurs particulièrement élevées en acides gras saturés à chaîne courte et moyenne (C8:0-C14:0), respectivement de $70 \%$ et $79 \%$, avec une prédominance de l'acide laurique (C12:0). Les acides palmitique (C16:0) et stéarique (C18:0) représentent environ 11 à $12 \%$, tandis que les acides gras (poly)insaturés sont minoritaires avec au plus $18 \%$ des acides gras totaux (huile de palmiste).

Les stéarines de palme, sont des fractions d'huile de palme à forte teneur en acides palmitique et stéarique $(50 \%$ à $80 \%$ ), le reste étant essentiellement constitué d'acides gras insaturés (C18:1, C18:2).

De par leur composition particulière, ces huiles et fractions trouvent applications dans de nombreux secteurs, au premier

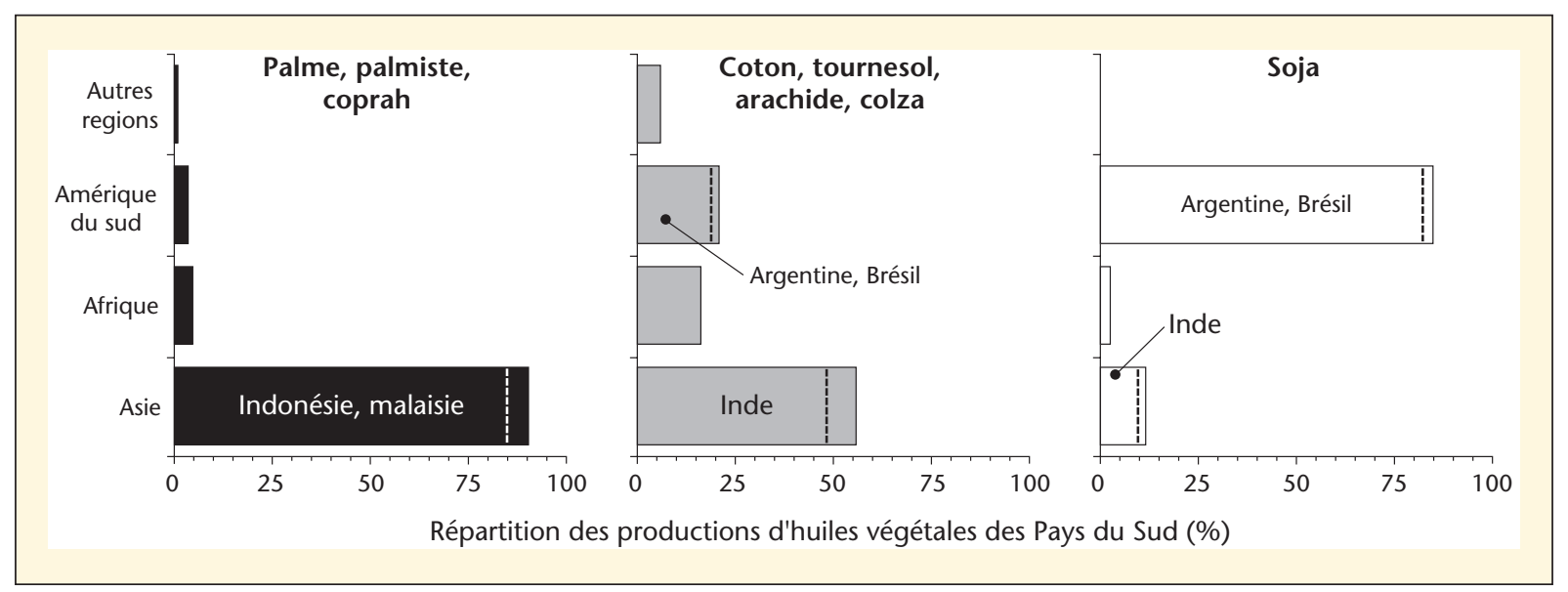

Figure 2. La production d'huiles végétales des Pays du Sud en 2011. Répartition géographique en fonction des types d'huiles. 
Tableau 1. Evolution de la part des usages non alimentaires dans la consommation mondiale d'huiles végétales.

\begin{tabular}{|llllll|}
\hline $\begin{array}{l}\text { Usages non alimentaires } \\
\text { (Part et type) }\end{array}$ & $\begin{array}{l}\text { 9 huiles } \\
\text { majeures }\end{array}$ & $\begin{array}{l}\text { Huile de } \\
\text { palme }\end{array}$ & $\begin{array}{l}\text { Huile de } \\
\text { coprah }\end{array}$ & $\begin{array}{l}\text { Huile de } \\
\text { colza }\end{array}$ & $\begin{array}{l}\text { autres huiles } \\
\text { (dont soja) }\end{array}$ \\
\hline Situation en 2001 (\%) & 14 & 16 & 41 & 9 & 10 \\
\hline Situation en 2011 (\%) & 25 & 26 & 46 & 32 & 90 \\
\hline Evolution sur 10 ans (\%) & 79 & 63 & 12 & 324 & $\checkmark \checkmark \checkmark \checkmark$ \\
\hline Carburant (biodiesel) & - & $\checkmark$ & $\checkmark$ & - & - \\
\hline Energie (combustible) & - & $\checkmark \checkmark$ & $\checkmark$ & $\checkmark \checkmark \checkmark$ & $\checkmark \checkmark \checkmark$ \\
\hline Chimie fine & - & $\checkmark \checkmark \checkmark$ & $\checkmark \checkmark \checkmark \checkmark$ & & $\checkmark$ \\
\hline
\end{tabular}

$\checkmark$ faible, $\checkmark \checkmark$ moyen, $\checkmark \checkmark \checkmark$ élevé, $\checkmark \checkmark \checkmark \checkmark$ très élevé.

rang desquels celui des détergents, sous forme de dérivés divers :

- glycérides partiels : solvants, humidifiants, stabilisants, lubrifiants, antigels ; - acides : parfums, adoucissants, plastifiants, bougies, cosmétiques, savons ; - esters: savons de haute qualité, détergents (forme sulphonatée) ;

- alcools : détergents sous forme sulphate ou éthoxylate ;

- ammoniums quaternaires : adoussicants (esterquats), agents de surface (imidazolines).

\section{Huile de soja et oléine de palme}

Comme évoqué plus haut, les huiles insaturées présentent un fort potentiel de fonctionnalisation. Hors biocarburants, la synthèse de dérivés époxydés est aujourd'hui une des applications industrielles majeures de I'huile de soja (environ 600000 tonnes en 2010) et, dans une moindre mesure, de palme. En tant que telles, les huiles époxydées sont d'excellents plastifiants (substituants des phtalates) et stabilisants des PVC et caoutchoucs synthétiques. Elles sont également utilisées comme additifs dans les peintures, colles et adhésifs. Leur traitement par des anhydrides d'acides en présence d'amines tertiaires, ou par les bisphénols $(A, F)$ et leurs dérivés (DGEBA, DGEBF), conduit à des résines époxy aux propriétés mécaniques remarquables (Tan, 2010). Sous forme hydroxylée elles peuvent être polymérisées avec des diisocyanates pour donner des polyuréthanes ou, après acrylation, être copolymérisées par voie radicalaire avec les acrylates.

\section{Huile de ricin}

Avec environ 420000 tonnes en 2011, la production d'huile de ricin (Ricinus communis) semble très modeste comparée aux autres huiles végétales. Elle n'en demeure pas moins essentielle puisqu'à ce jour elle est la seule source naturelle d'acide gras hydroxylé monoinsaturé disponible à l'échelle industrielle. L'Inde est loin le premier producteur mondial de graines de ricin avec $75 \%$ du marché en 2010-2011, suivie par la Chine (11\%) et le Brésil (6\%). Cependant elle détient près de $90 \%$ des exportations mondiales $d$ 'huile et dérivés (370 000 tonnes).

En termes de composition, I'huile de ricin contient près de $90 \%$ d'acide ricinoléique (12-hydroxy-9-cis-octadé cénoïque), 6-8 \% d'acides oléique et linoléique et $2 \%$ d'acides stéarique et palmitique. La structure de I'acide ricinoléique, associant une double liaison et un hydroxyle, lui confère (ainsi qu'à l'huile dont il est issu) des propriétés physico-chimiques uniques qui en font une molécule de choix pour la lipochimie. De fait, I'huile de ricin est largement utilisée comme lubrifiant, plastifiant, diluant pour polymères, composant pour mousses polyuréthanes, émollient cosmétique, et dispersant pour pigments et fillers.

Quant à I'acide ricinoléique et son ester méthylique, ils sont à la base de deux intermédiaires clés dans la synthèse de polyamides haute performance, l'acide undécylénique (undec-10-énoique) et I'acide sébacique (decanedioique). Le polyamide PA 11 Rilsan ${ }^{\mathbb{B}}$, dont Arkéma a l'exclusivité, est produit à partir du premier, tandis que les polyamides PA 410, PA 610, PA 1010 et PA 1012 sont synthétisés par plusieurs compagnies (DSM, Rhodia, Evonik Industries, BASF, Arkema) à partir du second. Notons que le segment des polyamides biosourcés (totalement ou en partie) est particulièrement dynamique, avec un taux de croissance annuel compris entre 15 et
$20 \%$. Par ailleurs, au cours de la transformation de l'acide ricinoléique et du ricinoléate de méthylique en acides sébacique et undécylénique respectivement, sont générés divers coproduits comme I'heptanal, I'heptanol, l'octanol, le glycérol ainsi que les acides octanol̈que, heptanoïque et 12hydroxy-stéarique. Tous sont utilisés dans des secteurs aussi variés que la parfumerie, les cosmétiques, la pharmacie ou les lubrifiants.

\section{Huiles végétales et lipochimie : challenges et perspectives}

L'augmentation de la population mondiale (9-10 milliards d'individus en 2050), du niveau de vie et des modes d'alimentation dans les principales économies émergentes (Chine, Inde) et à la raréfaction des ressources pétrolières devraient conduire dans les prochaines années à une augmentation drastique des besoins alimentaires et industriels en huiles et matières grasses naturelles. II devrait en résulter une compétition accrue au niveau de l'utilisation des terres arables, des usages alimentaires et non-alimentaires et entre les usages industriels eux-mêmes. Comment répondre à ce défi majeur sans sacrifier aux contraintes environnementales et sociétales actuelles et futures ? Nombre d'experts s'accordent sur le fait que l'augmentation des terres cultivées restera limitée et ne pourra à elle seule constituer une réponse suffisante. Le même constat pourrait s'appliquer aux rendements des principales cultures oléagineuses: proches de leur optimum dans les pays industrialisés, ils ne sauraient être significativement améliorés dans les autres, sans une 
irrigation efficace et un apport massif $d^{\prime}$ intrants avec, en corolaire, des problèmes environnementaux et de ressources en eau. Que dire enfin des OMG qui, à ce jour, ont davantage suscité la controverse qu'apporté la démonstration de leur légitimité, voire de leur utilité ?

S'il les solutions qui répondront efficacement à ce défi restent encore à inventer, deux pistes mériteraient d'être développées dès à présent. La première concerne la réduction des déchets et des pertes tout au long des filières, qui représentent entre la récolte au champ et le consommateur près de $50 \%$. La deuxième, plus indirecte, concerne le contrôle des prix et la limitation des phénomènes de spéculation sur les denrées alimentaires qui ont récemment démontré toute la perversité de leurs effets. D'ailleurs, cette régulation pourrait être efficacement associée à la substitution d'une partie des cultures de rente par des cultures vivrières.

Aussi, comment assurer dans ce futur fortement contraint, le développement de la lipochimie? Vraisemblablement par la mise en place de mesures complémentaires à court, moyen et long termes, parmi lesquelles on pourrait citer :

- le basculement progressif des biocarburants de première génération (dont les esters méthyliques d'acides gras) vers ceux de deuxième génération (éthanol, bio-huiles et bio-gaz, d'origine lignocellulosique, obtenus par voies fermentaires ou thermochimiques). II devrait ainsi se libérer des volumes considérables d'huiles végétales ;

- le développement de cultures dédiées d'oléagineux non alimentaires, adaptés aux zones à faibles ressources hydriques ou à sols dégradés, pour limiter la compétition avec les cultures alimentaires. Plusieurs candidats sont à l'étude dont les genres Cuphea (acides gras C10-C12), Lesquerella (acide lesquerolique), Vernonia (acide vernolique), Brassica et Crambe (acide erucique) et Vernicia (acides éleostéarique). De nombreux efforts au niveau de la sélection, de I'amélioration variétale, des pratiques culturales ou encore de la valorisation des coproduits (toxiques pour certains), sont nécessaires pour entrevoir un développement industriel de ces plantes, notamment dans les Pays du Sud. À ce titre, le Jatropha (Jatropha curcas) est un exemple d'échec particulièrement instructif. Souvent présenté comme "l'or vert du futur", les griefs qui sont faits à sa culture industrielle sont à la mesure des espoirs qu'elle a suscités : détournement de terres cultivables, faibles rendements, besoins élevés en eau, fertilisants et pesticides... Cependant, l'échec de nombreux projets de grande envergure (associant souvent des multinationales pétrolières) ne doit pas occulter tout l'intérêt d'une culture du Jatropha à l'échelle locale (sous forme de haies de séparation par exemple) notamment en termes d'autonomie énergétique ou de complément de revenu ;

- I'amélioration des procédés de transformation et la valorisation complète des matières premières en s'appuyant sur le potentiel de la bioraffinerie, des technologies blanches (génie microbiologique) et de la chimie verte. II faudra également répondre à l'évolution de la demande des différents secteurs de marché par un portefeuille de molécules et matériaux innovants, adaptés, et économiquement compétitifs.

\section{Conclusion}

$\mathrm{Au}$ cours de ces dernières années, la lipochimie a connu un essor particulièrement important pour des raisons techniques, énergétiques, environnementales, économiques et sociétales d'une part, et d'autre part, grâce à la disponibilité et à la diversité de matières premières renouvelables que sont les huiles végétales. II est donc naturel d'associer les Pays du Sud à ce succès puisque $58 \%$ de la production mondiale $d$ 'huiles végétales alimentaires (88 Mt) y sont réalisés, essentiellement en Asie (Malaisie, Indonésie, Inde) et en Amérique du Sud (Argentine, Brésil). La situation est cependant fort contrastée en termes de consommation: I'Inde utilise la totalité de sa production pour nourrir sa population. La Malaisie et I'Indonésie exportent près des trois quarts de leurs huiles de palme, palmiste et coprah, et en utilisent $15 \%$ pour des usages non-alimentaires, en chimie fine principalement. Quant à I'huile de soja produite au Brésil et en Argentine, $40 \%$ sont exportés, $27 \%$ sont destinés à I'alimentation et une part importante du tiers restant est convertie en biodiesel. Ainsi, les huiles de palme, palmiste, coprah et soja sont les huiles des Pays du Sud les plus concernées par le secteur de la lipochimie, auxquelles il convient d'ajouter I'huile de ricin, non alimentaire, dont l'importance tient plus à sa composition unique et à ses applications qu'à son volume de production $(0,42 \mathrm{Mt})$. Portée par les secteurs des biocarburants, de la chimie fine et des polymères, la part des usages industriels de ces huiles à considérablement augmenté ces dix dernières années, les champs d'applications, aussi nombreux que variés, étant dictés par leur composition en acides gras saturés (courts, moyens ou longs), insaturés (une ou plusieurs double liaison(s)) et fonctionnalisés (hydroxyles). Pourtant, malgré ce panorama plutôt flatteur, de nombreuses interrogations se posent quant à l'avenir de la lipochimie avec, en premier lieu, la question de la disponibilité des matières premières. En effet, le scénario d'une population avoisinant les 10 milliards d'êtres humains à l'horizon 2050 laisse présager une forte compétition entre usages alimentaires et non-alimentaires (et même entre usages non alimentaires) des huiles végétales, dans un contexte où la raréfaction des terres cultivables, les contraintes environnementales, la volatilité des prix et la spéculation financière seront autant d'obstacles à surmonter. Aussi, face à la complexité du défi à venir la réponse ne pourra être que multiple, intégrant certaines pistes évoquées dans cet article et d'autres qu'il reste encore à imaginer.

\section{Conflits d'intérêts : aucun}

\section{RÉFÉRENCES}

Dumeignil F. Propriétés et utilisation de I'huile de ricin. OCL $2012 ; 19$ : 10-5.

Gibbs FW. The History of the Manufacture of Soap. Annals of Science $1939 ; 4$ : 169-90.

Sherwood Taylor F. A history of industrial chemistry. New York : Abelard-Schuman, 1957.

Tan SG and Chow WS. Biobased epoxidized vegetable oils and its greener epoxy blends: $\mathrm{A}$ Review. Polymer-Plastics Technology and Engineering $2010 ; 49$ : 1581-90.

USDA-FAS Circular series - FOP 11-12 December 2011. 